\title{
MODELO DE TRANSFORMADORES EN SATURACIÓN UTILIZANDO FUNCIONES DE CÁLCULO DE PARÁMETROS EN EMTP-RV
}

\begin{abstract}
Resumen
La representación del fenómeno de saturación de un transformador es imprescindible en los estudios de transitorios electromagnéticos, principalmente porque debe representar con exactitud la sobrexcitación del transformador durante su energización, además de ser fundamental para estudiar casos de ferroresonancia (por ejemplo). En general, durante las pruebas de vacío que se aplican al transformador, se determina la corriente de excitación que circula por el núcleo ferromagnético a una determinada tensión. Sin embargo, cuando no exciten datos del fabricante con la curva de saturación del transformador, o las compañías eléctricas no tienen el equipo necesario para realizar este tipo de pruebas, es necesario utilizar otras alternativas. El presente documento hace una revisión de las funciones que se utilizan en el cálculo de parámetros para el modelado del transformador (incluyendo saturación), a partir de pruebas en campo. Estos modelos se encuentran presentes en la librería de elementos del programa de simulación de transitorios electromagnéticos EMTP-RV.
\end{abstract}

Palabras claves: ATP, BCTRAN, EMTP-RV, ferroresonancia, transformadores, transitorios electromagnéticos.

\begin{abstract}
The representation of the saturation phenomena in transformers is mandatory in electromagnetic transient analysis because it must accurately represent the transformer's over-excitation during energizing process, and being fundamental for ferroresonance analysis. In general, during transformer's open circuit test, the excitation current flowing through the ferromagnetic core for a certain voltage is calculated. Nonetheless, when manufacturers do not provide saturation curves or utilities do not have the necessary equipment to perform this test, it is necessary to use other alternatives. This paper reviews the data calculation functions used in order to model the transformer (including saturation), from field tests. These models are presented in the common library of the electromagnetic transient simulation program EMTP-RV.
\end{abstract}

Key words: ATP, BCTRAN, EMTP-RV, ferroresonance, transformers, electromagnetic transients.

Recibido: 15 de noviembre de 2012 • Aprobado: 2014

\section{INTRODUCCIÓN}

Durante las pruebas de vacío que se realizan a los transformadores, se determina la corriente de excitación que circula en el transformador para una determinada tensión. Una vez obtenido el valor eficaz de estos valores, se puede trazar la curva de saturación. Generalmente, en campo (subestaciones de $230 \mathrm{kV}$ o $138 \mathrm{kV}$ ) no es posible aplicar tensiones superiores al 110\% de la tensión nominal del devanado de prueba del transformador durante las pruebas en vacío debido al riesgo de daño(Dután, 2010), por lo que es común aplicar tensiones en un rango típico de $90 \%$ a $110 \%$, en pasos de $5 \%$, para así obtener valores de corriente de excitación. El problema radica en que aún si las mediciones logran captar algún grado de corriente de excitación, en condición de energización o 
mismo en estado de ferroresonancia, el valor de tensión de $110 \%$ se sobrepasa fácilmente, por lo que se vuelve necesario tener una curva de saturación con valores por encima del $110 \%$ de la tensión nominal, para una adecuada representación delnúcleo del transformador.

En la práctica programas como EMTPRV(Mahseredjian, Dennetière, Dubé, Khodabakhchian, \& Gérin-Lajoie, 2007), o de forma general de tipo EMTP, realizan la determinación tradicional de las condiciones de saturación por encima del último valor de tensión, mediante una extrapolación lineal de los dos últimos puntos de dicha curva(Dután, 2010). Por otro lado, los programas de tipo EMTP como el ATP(Dután, 2010)tienen, en general, dos formas de manejar los datos de tensión eficaz y corriente eficaz, obtenidos a partir de las pruebas, para convertirlos en una curva característica de flujo vs corriente $\left(\lambda_{\text {pico, }}, I_{\text {pico }}\right)$, ya sea empleando rutinas de cálculo de parámetros de transformadores, tales como SATURA(Mork, Gonzalez, \& Ishchenko, 2007), o mediante métodos más avanzados que pueden tomar en cuenta el acoplamiento trifásico de transformadores (Narang \& Brierley, 1994).

\section{EMTP: PROGRAMA DE SIMULACIÓN DE TRANSITORIOS ELECTROMAGNÉTICOS}

Un programa de transitorios electromagnéticos se utiliza para el cálculo de transitorios en sistemas de potencia, para el cálculo de transitorios en circuitos eléctricos y para aplicaciones en sistemas que no contemplen tiempo real(Dommel, 1969).Nótese que se define un transitorio electromagnético como un cambio súbito en las condiciones del sistema, provocado por la inadecuada interacción entre las partes inductivas y capacitivas de unared eléctrica. Estos pueden ser provocados por causas externas a la red eléctrica (descargas atmosféricas) o por causas internas (energización de líneas de transmisión).Este tipo de programas permiten el desarrollo de simulaciones y análisis de transitorios electromagnéticos para coordinación de protecciones, coordinación de aislamiento, diseño y dimensionamiento de redes de alta y media tensión, entre otros.
Haciendo un recorrido histórico en el desarrollo de este tipo de aplicaciones, se tiene que el primer programa de simulación de transitorios electromagnéticos EMTP formó parte de un programa de investigación de la Universidad de Columbia Británica en Vancouver, Canadá(Dommel, 1969). Se desarrolló como contraparte del programa llamado Analizador de transitorios en redes (TNA, por sus siglas en inglés), a finales de los años sesenta por Hermann W. Dommel (Meyer, Jerosolimski, \&Stubbe, 1998). Mahseredjian (2010) menciona que dicho programa fue punta de lanza de muchos desarrollos en el campo de los transitorios electromagnéticos, tales comoel programa de simulación de transitorios electromagnéticos en corriente continua (EMTDC, por sus siglas en inglés) o el programa alternativo de simulación de transitorios electromagnéticos (ATP, por sus siglas en inglés).

Mahseredjian (2010) señala que a finales de la década de los noventa y bajo su liderazgo, se creó un grupo de trabajo (DevelopmentCoordinationGroup, DCG-EMTP) cuya tarea fue la de reestructurar completamente la vieja versión del EMTP y crear una versión completamente nueva llamada EMTPRV. Asimismo, Mahseredjian (2010) indica que luego de cinco años de investigación y desarrollo, la primera versión comercial del EMTP-RV se lanzó en 2003, con alrededor de un millón de líneas de código.

\section{DEPENDENCIA FRECUENCIAL EN EL MODELO DE TRANSFORMADORES DE POTENCIA}

Los modelos de transformadores que se utilizan normalmente para la simulación de transitorios electromagnéticos, tienen diferentes niveles de detalle dependiendo de la aplicación para la cual se deseen utilizar. Sin embargo ningún modelo está en capacidad de modelar todos los fenómenos transitorios para todo el espectro de frecuencias posible. Mork et al. (2007)señalan que los rangos de frecuencia se pueden clasificar en cuatro grupos con algún grado de traslape entre ellos, tal y como lo muestra en el Cuadro 1.

A manera de ejemplo, una representación lineal simplificada de un transformador monofásico o trifásico puede implementarse 
Tabla 1. Modelado de transformadores de potencia.

\begin{tabular}{lllll}
\hline Parámetro & $\begin{array}{l}\text { Transitorios a baja } \\
\text { frecuencia }\end{array}$ & $\begin{array}{l}\text { Transitorios de } \\
\text { frente lento }\end{array}$ & $\begin{array}{l}\text { Transitorios de } \\
\text { frente rápido }\end{array}$ & $\begin{array}{l}\text { Transitorios de } \\
\text { frente muy rápido }\end{array}$ \\
\hline $\begin{array}{l}\text { Impedancia de corto } \\
\text { circuito }\end{array}$ & Muy importante & Muy importante & Importante & Despreciable \\
Saturación & Muy importante & Muy importante(1) & Despreciable & Despreciable \\
Pérdidas en el hierro & Importante(2) & Importante & Despreciable & Despreciable \\
Corriente de Eddy & Muy importante & Importante & Despreciable & Despreciable \\
Acople capacitivo & Despreciable & Importante & Muy importante & Muy importante \\
\hline
\end{tabular}

Fuente: Mork et al (2007).

(1) Solo para fenómenos de energización, sino simplemente es importante.

(2) Solo para fenómenos de resonancia.

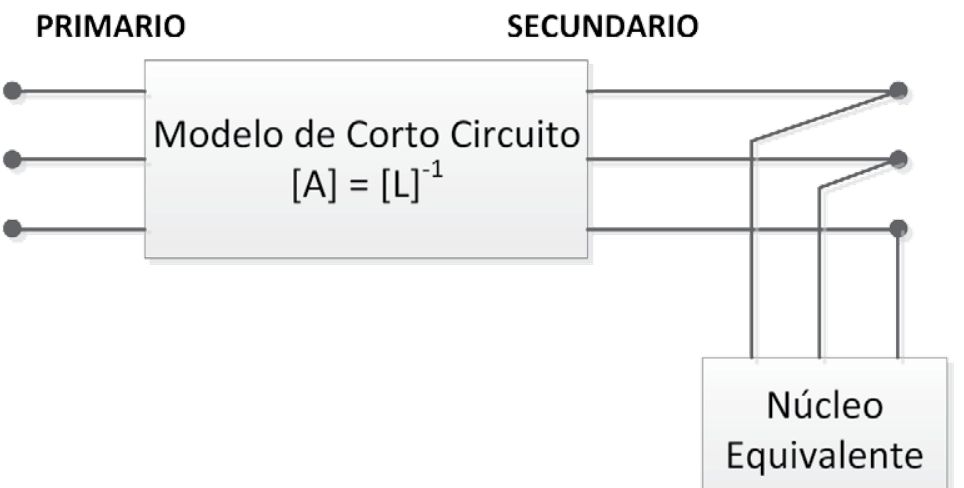

Figura 1. Conceptualización de la combinación de la matriz inversa de inductancias con rama de magnetización adjuntada externamente para un transformador de dos devanados.

Fuente: Mork et al (2007).

en el EMTP en la forma de un impedancia en serie o utilizando matrices de admitancias, a partir de la rutina BCTRAN(Mork et al., 2007). Sin embargo este enfoque no incluye topologías con un núcleo de varias columnas (por ejemplo transformadores de más de dos devanados) o los efectos no lineales en el núcleo de hierro. Como se indicó inicialmente, para análisis transitorio de ferroresonancia, corriente de energización y otros comportamientos no lineales, es necesario considerar los efectos de saturación, aún si en algunos casos la rama de excitación se adjunta externamente al modelo en la forma de elementos no lineales similar a lo expuesto en la Figura 1.

A pesar de que Mork et al. (2007) definen como una buena práctica colocar el equivalente del núcleo al exterior del modelo del transformador, en realidad existe una serie de limitaciones, que entre las más importantes se encuentra: posible inestabilidad numérica (observable principalmente en trasformadores de tres devanados), utilidad limitada para unidades trifásicas, y conexiones 
topológicamente incorrectas (posibles en simulación pero no en la realidad), atentando con el correcto comportamiento del modelo ante fenómenos electromagnéticos.

Además, modelos detallados que incorporan ciertas no linealidades dentro del modelo del núcleo del transformador utilizan el principio de dualidad(Mork et al., 2007). En este caso el modelo incluye los efectos propios de la saturación para cada columna del núcleo, efectos de fuga o dispersión magnética y acoplamiento magnético. Estos modelos se basan en el desarrollo de parámetros concentrados a partir del circuito magnético equivalente, es decir, se observan las longitudes de los caminos magnéticos en las columnas (utilizando reluctancias), para así representar los flujos magnéticos dentro del núcleo del transformador(Mork et al., 2007). Finalmente estos modelos permiten la conversión subsecuente del circuito magnético al circuito eléctrico equivalente usando transformaciones duales o lo que se conoce como el principio de dualidad (Mork et al., 2007).

\section{MODELO DE TRANSFORMADORES PARA EMTP-RV INCLUYENDO SATURACIÓN}

Como se mencionó anteriormente, en programas de tipo EMTP, el modelado del núcleo del transformador se basa en la curva

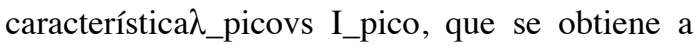
partir de los datos de tensión y corriente eficaz de la prueba de vacío mediante la rutina SATURA, la cual realiza una extrapolación lineal de los dos últimos puntos de datos, considerando constante la pendiente del segmento formado por dichos puntos(Dután, 2010). Además SATURA asume que la variación del flujo magnético es sinusoidal a la frecuencia fundamental como una función del tiempo. Igualmente asume que:

- Las pérdidas debidas a corrientes parásitas e histéresis se pueden despreciar.

- Las resistencias de los devanados se pueden despreciar.

- La curva característica $\lambda_{\text {pico }}$ vs $I_{\text {pico }}$ se genera punto por punto, realizando una interpolación lineal.
Nótese que en la mayoría de estas funciones, una vez que se utilizan para, a partir de datos de prueba, calcular el circuito equivalente del transformador, el circuito resultante se implementa como un circuito multifásico acoplado RL (resistencia e inductancia en serie).

\subsection{Modelo BCTRAN}

El modelo BCTRAN es un modelo desarrollado luego que un primer modelo llamado Saturable (desarrollado en ATP), el cual presentaba grandes limitaciones al momento de modelar transformadores trifásicos(Narang \& Brierley, 1994). El modelo Saturable incorporaba en su entrada de datos una tabla en la cual se ingresaba los valores eficaces de tensión y corriente de la prueba en vacío y el programa ATP convertía estos datos en una curva característica $\lambda_{\text {pico }}$ vs $I_{\text {pico }}$ mediante la rutina SATURA, y así se representaba el núcleo conectándolo finalmente al primario del transformador(Dután, 2010). Este modelo consideraba a sus elementos como desacoplados y era ampliamente usado para representar transformadores monofásicos, mientras que no tuvo la misma aceptación para transformadores trifásicos(Dután, 2010).

Por lo anterior, se desarrolló el modelo BCTRAN, el cual es una representación lineal simplificada de transformadores (monofásicos o trifásicos) en la forma de matrices de impedancias o admitancias en derivación, tomando en cuenta los acoplamientos mutuos entre devanados(Dután, 2010). Este tipo de modelo conecta externamente el modelo equivalente del núcleo a partir de la información recabada en la rutina BCTRAN en forma de curva de saturación que toma en cuenta el lazo de histéresis, tal y como lo muestra la Figura 2.

Por otro lado, la función para el cálculo de parámetros BCTRAN presenta que, para un transformador trifásico con un núcleo de tres columnas o piernas, la corriente de excitación para una prueba de secuencia homopolar es relativamente alta y no puede ser despreciada. Así, la admitancia en derivación de la rama de magnetización, la cual se agrega al modelo para representar la corriente de excitación y las pérdidas por excitación, se calcula entonces a partir de los 


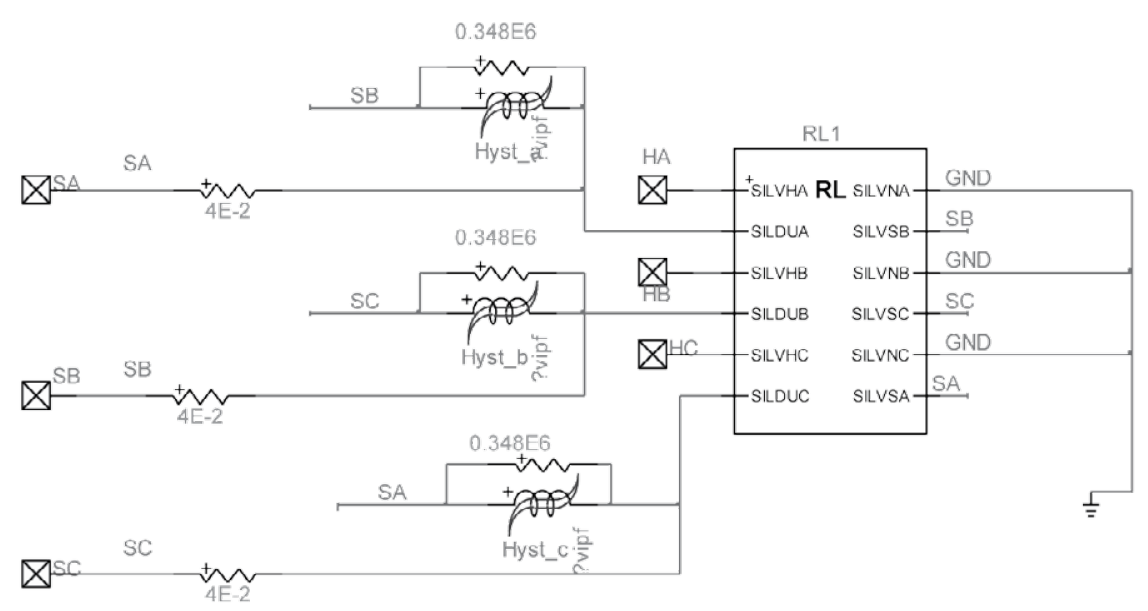

Figura 2. Conexión externa del modelo de núcleo del transformador al elemento BCTRAN.

datos de la prueba de excitación(Dommel, 1986). Hay dos tipos de valores: uno para las pruebas de secuencia positiva y otro para las pruebas de secuencia homopolar, que se convierten posteriormente en una submatriz de $3 \times 3$ (Dommel, 1986). Finalmente para completar el modelo, es requerido igualmente los datos de la prueba de cortocircuito realizada al transformador.

\subsection{Modelo TRELEG}

Los efectos de un núcleo de tres columnas en el modelado de un transformador son aparentes en los diferentes valores determinados a partir de las impedancias de cortocircuito en secuencia positiva y homopolar. Nótese que el circuito equivalente de secuencia homopolar del transformador depende del grupo de conexión del transformador, la puesta a tierra o no del neutro (rígidamente puesto a tierra o aislado en conexiones estrella) y del tipo de núcleo magnético (tres columnas, cinco columnas, acorazado o bancos monofásicos de transformación).

Al igual que en BCTRAN, estos valores son utilizados por el programa para calcular un modelo basado en una matriz de impedancias de $3 n \times 3 n$, donde $n$ es el número de devanados del
transformador(Dommel, 1986). De igual manera, Dommel (1986) señala que se debe asumir que los devanados están concéntricamente localizados en el núcleo y se ingresan en el orden del devanado exterior al interior. Cuando los devanados no son concéntricos, o cuando los devanados en delta no están en el interior del núcleo, el programa provee una opción para retener los devanados con conexión delta como los últimos devanados, y así proporcionar la impedancia de magnetización para cada devanado(Dommel, 1986).

Así mismo Dommel (1986) indica que en ausencia de cualquier tipo de dato de prueba, la impedancia de magnetización de secuencia positiva de los devanados (en por unidad) puede asumirse como que aumenta conforme aumenta el diámetro, mientras que la impedancia de magnetización de secuencia homopolar decrece. Igualmente, señala que las variaciones de un devanado con respecto al otro serán aproximadamente iguales a la reactancia de cortocircuito de secuencia positiva entre los mismos. No aparece registrado en artículos posteriores a la implementación de este modelo, que las aproximaciones de las impedancias de magnetización tengan alguna repercusión negativa en la veracidad del modelo. 


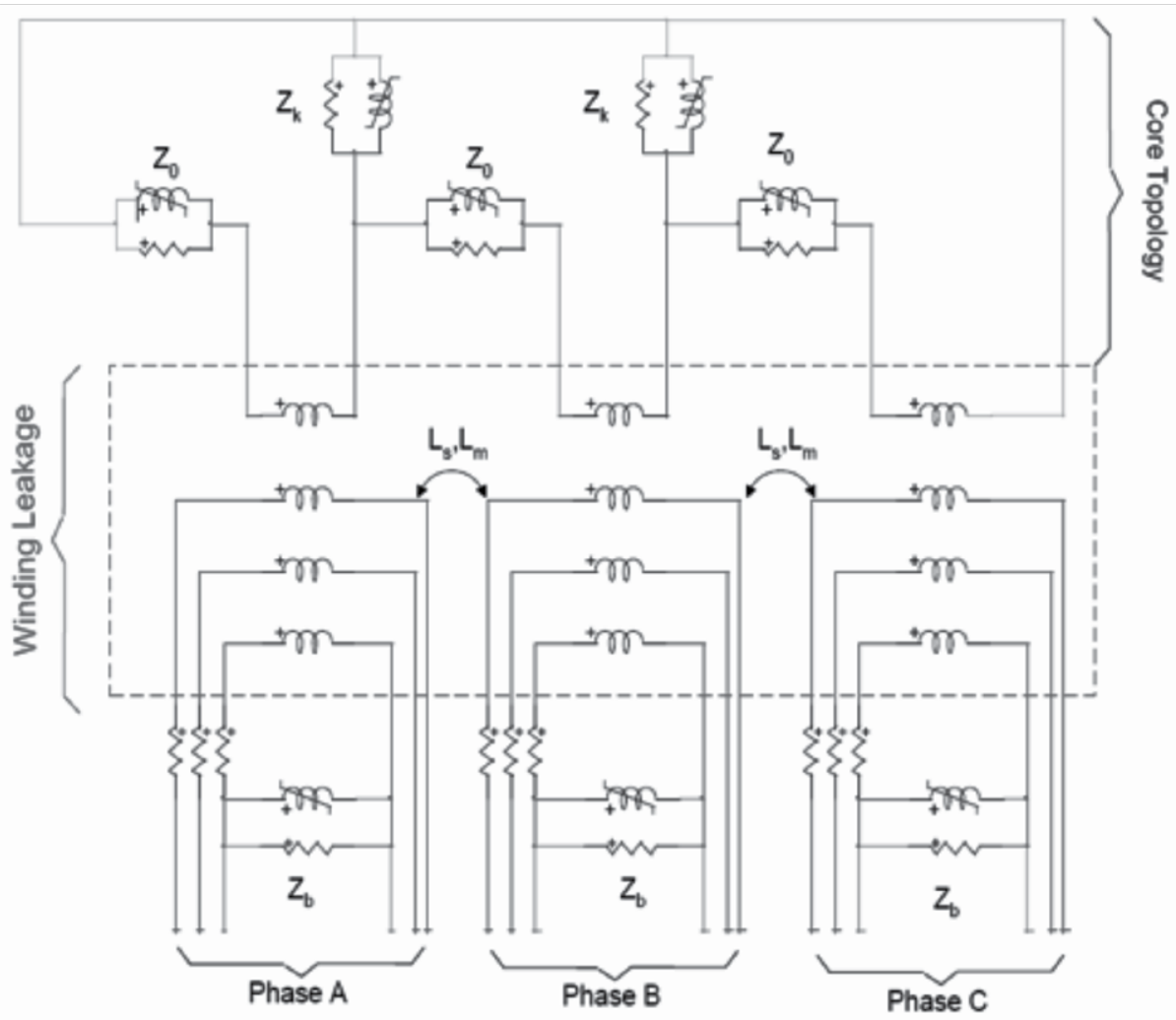

Figura 3. Representación de un modelo de transformador trifásico con núcleo de tres columnas. Fuente: EMTP-RV Versión 2.3.0 (2012).

\subsection{Modelo TROPMAG}

Conceptualmente este modelo toma en cuenta cualquier topología de un circuito magnético formado por un núcleo y sus devanados, para así ensamblar una representación basada en un circuito eléctrico equivalente tal y como lo muestra la Figura 3. Para unidades con tres o cinco columnas, cada columnaes modelada individualmente y se agrega a una matriz de admitancias reproduciendo el correcto acoplamiento magnético entre devanados (EMTP-RV Version 2.3.0, 2012).Un tercer devanado adicional (denominado devanado ficticio) es necesario para establecer dicha interfaz, ya que las columnas del núcleo están aisladas eléctricamente de los devanados (EMTPRV Version 2.3.0, 2012).

Las salidas de esta rutina consisten en la siguiente información (de donde $\mathrm{n}$ es el número de devanados):
- Matriz simétrica de admitancias de hasta orden 3(n+1), dependiendo del tipo de núcleo y de las opciones específicas de modelado (EMTP-RV Version 2.3.0, 2012).

- Una red de ramales en paralelo de tipo RL que modelan las corrientes de magnetización debido a alguna columna bobinada, yugos horizontales, o el camino de retorno de secuencia homopolar a través del aire para transformadores de núcleo de tres columnas o bien a través de las columnas externas en transformadores de núcleo de cinco columnas (EMTP-RV Version 2.3.0, 2012).

Así mismo, la rutina requiere una cantidad de valores de prueba de cortocircuito de secuencia homopolar del orden de $n(n-1) / 2$ para n devanados de un núcleo de cinco columnas o para transformadores monofásicos (EMTP-RV Version 2.3.0, 2012). Para transformadores con núcleo con tres devanados, al 


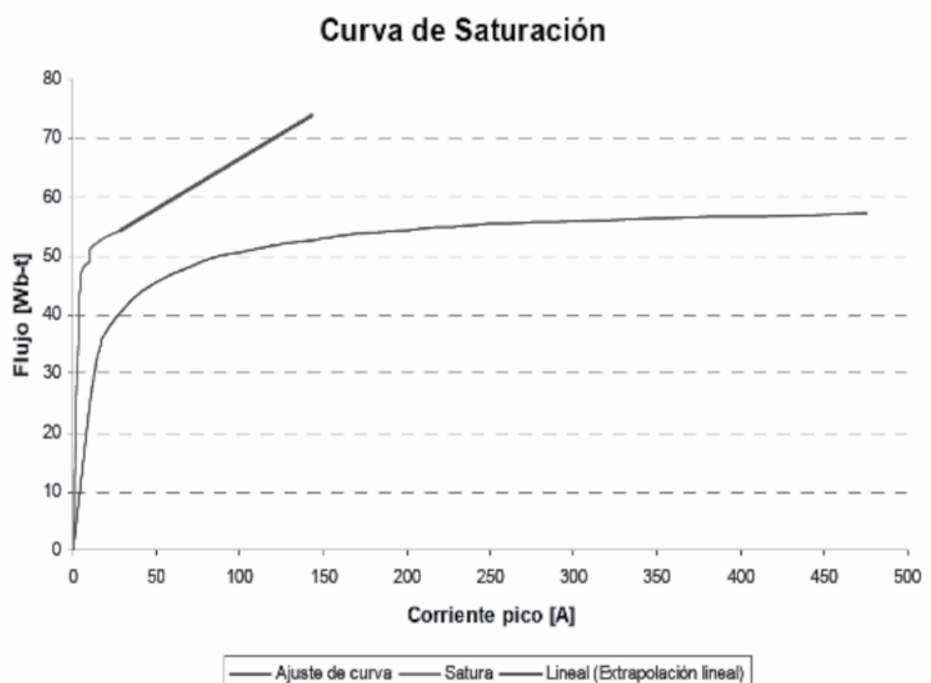

Figura 4. Extensiones de la curva de saturación a partir del ensayo en vacío usando la subrutina SATURA y un ajuste de curvas.

Fuente: Durán (2010).

menos un parámetro adicional es necesario para caracterizar el rendimiento en secuencia homopolar. Este parámetro adicional puede ser:

- Corriente de excitación de secuencia homopolar.

- Unaprueba para determinar la impedancia de cortocircuito de secuencia homopolar, ejecutada de manera que la excitación esté en el devanado 1 y cualquier otro devanado $m$ esté cortocircuitado, de forma tal que ningún otro devanado esté conectado en delta durante el ensayo (EMTP-RV Version 2.3.0, 2012).

En resumen, esta rutina requiere la siguiente información eléctrica: datos de excitación, datos de los devanados (identificados y caracterizados por el usuario) y datos del ensayo de cortocircuito (EMTP-RV Version 2.3.0, 2012).

\section{NUEVAS APROXIMACIONES: MODELOS HÍBRIDOS}

A inicios de la década de los noventa el grupo de usuarios de ATP en Brasil (Dután, 2010) notó que la extrapolación lineal que utiliza la subrutina
SATURA fallaba en algunas casos, debido a que en el ensayo en vacío, el transformador no alcanza la saturación completa para niveles de excitación de hasta $110 \%$ de la tensión nominal, y la extensión lineal (que ejecuta la extrapolación) de sus dos últimos puntos causa un severo error (en los casos detectados por el grupo de usuarios) en la determinación de la corriente de magnetización para cualquier nivel de excitación por encima del último punto especificado (Dután, 2010). La Figura 4 muestra la diferencia entre la extrapolación de la curva de saturación usando la subrutina SATURA y un nuevo método (Mork et al., 2007) basado en un ajuste de curvas.

Así, tomando en cuenta lo expuesto anteriormente, se desarrolló un modelo híbrido basado en el mejoramiento del modelo BCTRAN (Mork et al., 2007). Dután (2010) señala que estos esfuerzos iban dirigidos a:

- Usar la matriz [A] para una representación completa de las inductancias de dispersión, agregando los acoples magnéticos entre los devanados.

- Usar una representación topológicamente correcta de la no linealidad del núcleo basada en el principio de dualidad. 


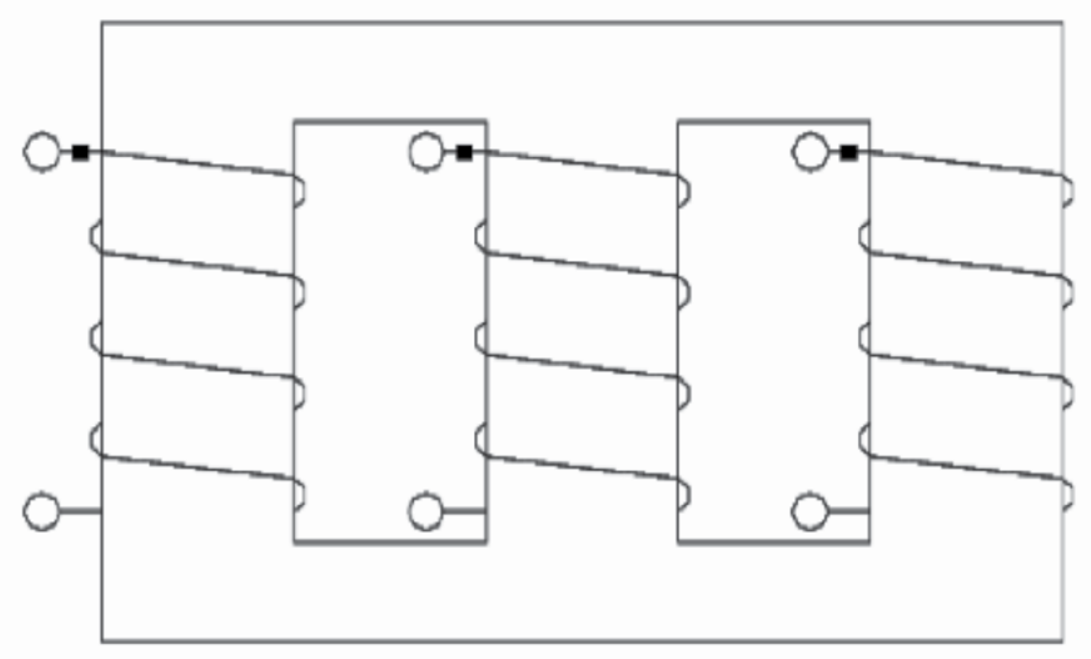

Figura 5. Núcleo de tres columnas. Por simplicidad se muestra solo un conjunto de devanados.

Fuente: Mork, et al (2007).

- Utilizar resistencias en los devanados dependientes de la frecuencia.

- Incluir los efectos capacitivos en el modelo.

\subsection{Representación del núcleo basado en el principio de dualidad}

Un transformador conectado en estrelladelta con un núcleo de tres columnas se utiliza para ilustrar este principio. La Figura 5 y Figura 6 muestran la estructura física y el correspondiente circuito magnético concentrado. Nótese que la letra $R$ representa la reluctancia magnética. Mork et al. (2007) señalan que la derivación completa de este modelo está basada en el principio de dualidad: los nodos del circuito magnético se convierten en mallas o bucles cerrados en el circuito eléctrico, mientras que las mallas del circuito magnético se convierten en nodos del circuito eléctrico.

En la Figura 6, $R_{L}$ representa el camino del flujo a través de las columnas del núcleo del transformador. $R_{Y}$ representa el camino del flujo a través de los yugos, $R_{2}$ representa el camino de fuga entre las columnas y los devanados más internos (Mork et al., 2007). De igual forma, Mork et al. (2007) indican que se deben representar los caminos de fuga entre las columnas y los espacios entre las dos bobinas $\left(R_{3}\right)$ y entre las columnas y el devanado más externo $\left(R_{4}\right)$. Finalmente, $R_{p h}$ representa el camino de flujo de dispersión a través del aire en paralelo con el yugo. El resultado, son cuatro partes distintas y funcionales resumidas en un circuito equivalente el cual se puede encontrar en Mork et al. (2007):

- Representación de las inductancias de fuga o dispersión.

- Representación topológicamente correcta del núcleo

- Resistencias de los devanados.

- Efectos capacitivos.

\subsection{No linealidad del núcleo}

Mork et al. (2007) señalan que el modelo del núcleo, basado en el principio de la dualidad, se conecta a través de un devanado ficticio $\mathrm{n}+1$, donde $\mathrm{n}$ es el número de devanados del transformador. Este devanado ficticio funciona como punto de conexión del núcleo equivalente, tal como lo muestra la Figura 7, para un núcleo de tres columnas. La correcta representación topológica del núcleo no lineal es extraída del principio de dualidad y conectada a las terminales $\alpha-\beta-\gamma$ del devanado $n+1$ en la Figura 7 . 


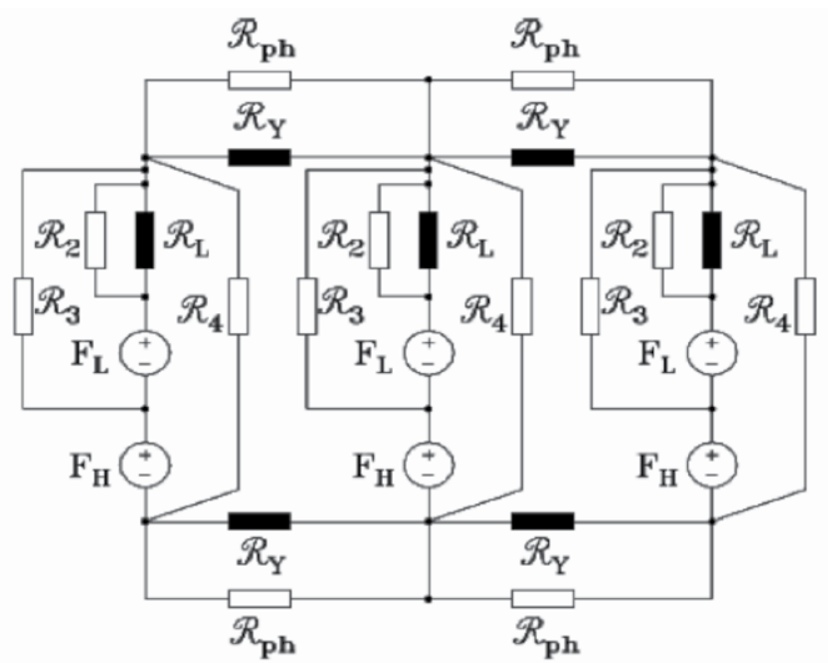

Figura 6. Circuito magnético. Reluctancias representan los cambios del flujo magnético. Fuente: Mork, et al (2007).

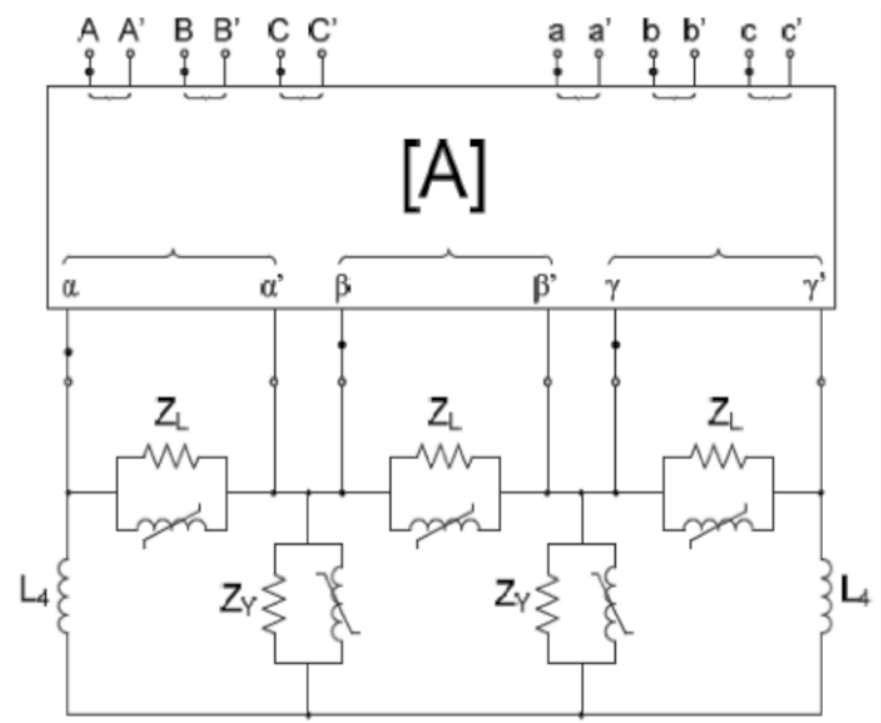

Figura 7. Núcleo conectado al devanado ficticio $n+1$.

Fuente: Mork, et al (2007).

Mork et al. (2007) establecen que cada columna y yugo es representado por una resistencia de pérdidas en el núcleo junto con una inductancia saturable. Así mismo, los caminos del flujo de secuencia homopolar se representan por las inductancias $L_{4}$. Esta representación RL de cada columna del núcleo es satisfactoria en muchos casos. Nótese que si se dispone de los datos de diseño, el equivalente del núcleo puede ser determinado basándose en el cálculo de la curva de saturación $\lambda$ vs $i$ para cada columna del núcleo. Por lo anterior, Mork et al. (2007) indican que la estimación de la curva de saturación es más real debido a que el tipo de material es conocido, así 
como también el área de sección transversal del núcleo y el número de vueltas de los devanados. Nótese además que el tipo de material define la curva de flujo magnético $(B)$ en función de la intensidad magnética $(H)$ (conocida como la curva $B-H$ ), la cual es la misma para todas las componentes del núcleo y puede ser aproximada por la ecuación de Fröhlich (en algunos textos traducidos al castellano se encuentra como Frölich). Esta ecuación proporciona una curva suave en función de dos parámetros $a$ y $b$, y se muestra en la Ecuación (1).

$B=H /((a+b) \cdot|H|)$

La curva $\lambda$ vs i se puede estimar por medio del escalamiento de la curva B-H, de acuerdo con las áreas y longitudes de los componentes del núcleo. Así mismo, Mork et al. (2007) establecen que si las dimensiones de las columnas y yugos son desconocidas, se pueden utilizar relaciones normalizadas en función de las dimensiones del núcleo. Si por el contrario se desconocen las dimensiones del núcleo, estas pueden ser asumidas en aquellos casos donde las relaciones típicas puedan ser usadas sin que se introduzca un error considerable. Esto porque las relaciones entre las dimensiones del núcleo varían en un pequeño rango de acuerdo a normas de diseño. Por lo tanto, la ecuación de Fröhlich es conveniente para representar la curva de magnetización del núcleo.

\section{ESTRUCTURA DEL MODELO HÍBRIDO XFMR DESARROLLADO PARA ATP}

Tomando como base el modelo híbrido expuesto anteriormente, los desarrolladores del programa ATP crearon el modelo XFMR (Dután, 2010), el cual sirve para simular transformadores trifásicos de dos o tres devanados, así como autotransformadores, en acoplamientos estrelladelta (Dután, 2010).

El modelo incluye una matriz de inductancia inversa para la descripción de los flujos de dispersión. Además, de forma opcional se puede modelar las resistencias de los devanados y su respectiva dependencia frecuencial, acoplamientos capacitivos y un modelo topológicamente correcto del núcleo con saturación individual y pérdidas en columnas y yugo (Mork et al., 2007). Por lo tanto, se puede simular el transformador basado en tres fuentes de datos:

- Datos de diseño (especificación geométrica y parámetros del material del núcleo y devanados).

- Reporte de pruebas (similar al modelo BCTRAN excepto para el modelo del núcleo).

- Valores típicos (basados en tensiones y potencias nominales).

Así, el núcleo se conecta a un devanado ficticio en el exterior y las características de la magnetización se obtienen en referencia a la ecuación Fröhlich (Dután, 2010). Ahora bien, para establecer el devanado ficticio del núcleo, se debe especificar la secuencia de los devanados a partir del núcleo.Dután (2010) establece que el punto de conexión de los devanados del núcleo se constituye basándose en el camino de fuga entre el devanado más interno y el núcleo.

Por otro lado, las partes inductivas y resistivas del núcleo son tratadas independientemente, mientras que las pérdidas en el núcleo son asumidas como lineales, por lo que cada parte del núcleo es modelada con su propia resistencia de pérdidas. Así, la esencia de modelar el núcleo inductivo radica en obtener los valores $a^{\prime}$ y $b^{\prime}$ en la ecuación de Fröhlich a partir de los cuales se llega a determinar la característica de saturación $\lambda$ vs i mediante una técnica de optimización (Dután, 2010). El valor eficaz medido o asumido de la corriente de magnetización se compara con una cantidad calculada e iterada hasta que se logra el mejor ajuste.

Como resultado de la implementación de la ecuación de Fröhlich, la relación flujo corriente ( $\lambda$ vs $i$ ) se define como:

$i=l_{r} \cdot\left(a^{\prime} \cdot\left(N A_{r}\right)\right) /\left(1-\left(b^{\prime} \cdot N A_{r}\right)\right)$

donde $l_{r}$ y $A_{r}$ son la longitud y sección relativa del núcleo relacionado con la columna (Dután, 2010). Las constantes a determinar en el proceso de optimización vienen dadas por (3) y (4):

$a^{\prime}=a \cdot\left(l_{L} /\left(N^{2} \cdot A_{L}\right)\right)$ 
$b^{\prime}=b \cdot\left(1 /\left(N^{2} \cdot A_{L}\right)\right)$

Las ecuaciones anteriores se basan en la longitud y sección transversal absolutas de la columna del núcleo. El resultado final indica que para el cálculo del flujo por la columna se tiene que (Dután, 2010):

$\lambda_{L}=i /\left(\left(a^{\prime}+b^{\prime}\right) \cdot i\right)$

Mientras que para el flujo fuera de la columna se tiene entonces que (Dután, 2010):

$\lambda_{0}=\left(i \cdot A_{r 0}\right) /\left(a^{\prime} \cdot I_{r 0}+b^{\prime} \cdot i\right)$

Finalmente, Dután (2010) señala que el flujo que atraviesa el yugo está dado por:

$\lambda_{L}=\left(i \cdot A_{r y}\right) /\left(a^{\prime} \cdot I_{r y}+b^{\prime} \cdot i\right)$

Si un único punto del ensayo en vacío se ingresa en el modelo XFMR, el núcleo se asume como lineal, mientras que si se especifica más de un punto, estos son enviados a la rutina de optimización la cual retorna los valores de $a^{\prime}$ y $b^{\prime}$ usados para describir la relación $\lambda$ vs $i$ en el núcleo (Dután, 2010).

Por otro lado, cuando se utiliza la rutina SATURA, como se explicó anteriormente, el usuario debe ingresar los valores eficaces medidos de la curva de tensión vs corriente para una secuencia de puntos, asumiendo una interpolación lineal entre ellos. Como resultado, la salida de esta interpolación es una curva $\lambda_{\text {pico }}$ vs $I_{\text {pico }}$ lineal a trozos. En general, los datos de la prueba a vacío vienen dados por valores eficaces de línea, mientras que la rutina SATURA requiere como datos de ingreso los valores de tensión y corriente de fase, por lo que cuando se tienen conexiones en delta se debe tener una consideración especial (Dután, 2010).

De forma general se asume que para una conexión en delta la corriente de fase es igual a la corriente de línea dividida por $\sqrt{3}$, sin embargo, esta hipótesis es un tanto dudosa cuando el transformador opera en condición de saturación, ya que en ese momento las corrientes no son sinusoidales (Dután, 2010).

Por lo anterior, Nicola Chiesa desarrolló otra subrutina llamada "rms2peak" (Dután, 2010), que solucionaba este problema evitando la conversión de la corriente de línea en su valor de fase. La subrutina rms2peak puede ser resumida en tres pasos:

- Conversión de las tensiones eficaces de línea en valores pico de flujo de concatenación para todos los puntos de la curva característica $V_{r m s}$ vs $I_{r m s}$.

- Estimación de la corriente pico del primer punto de la característica de magnetización.

- Cálculo de los restantes puntos de la característica de magnetización con un método numérico iterativo que ajusta cada pico de corriente.

\section{CONCLUSIONES}

Ciertas limitaciones presentes durante el modelado de transformadores en condiciones de saturación han sido expuestas en este documento. Programas de tipo EMTP, como el EMTPRV o el ATP, proveen al usuario de una serie de rutinas para determinar la característica de magnetización y adjuntarla dentro del modelo del transformador, ya sea a partir de ensayos en vacío o bien mediante datos del fabricante o pruebas de cortocircuito para determinar la impedancia de secuencia directa y/o homopolar. Es importante recalcar que la mayoría de estas rutinas utilizan métodos de iteración o ajuste de curvas que, para algunos casos, ocasionan severos errores en la determinación de la curva de flujo vs corriente, y por lo tanto, pueden afectar la veracidad de las simulaciones cuando se requieren hacer estudios de transitorios durante la energización del transformador o bien durante condiciones de operación en presencia de ferroresonancia.

\section{REFERENCIAS BIBLIOGRÁFICAS}

Dommel, H. W. (1969). Digital Computer Solution of Electromagnetic Transients in Single-and Multiphase Networks. IEEE Trans. on Power Apparatus and Systems, PAS-88(4), 388-399.

Dommel, H. W. (1986). Electromagnetic Transients Program Theory Book. (T. Liu $\&$ W. Scott Meyer, Eds.) (1st ed.). Portland, USA: Bonneville Power Administration. 
Dután, L. (2010). Modelación de Transformadores para Estudios de Energización usando ATPEMTP (pp. 1-9). Quito, Ecuador.

EMTP-RV Version 2.3.0. User Guide: Transformer Data TOPMAG.

Mahseredjian, J. (2010). Notas del Curso ELE8457 Comportement des Réseaux Électrique. Montreal, Quebec, Canadá.

Mahseredjian, J., Dennetière, S., Dubé, L., Khodabakhchian, B., \& Gérin-Lajoie, L. (2007). On a new approach for the simulation of transients in power systems. Electric Power Systems Research, 77(11), 1514-1520.

Meyer, B., Jerosolimski, M., \& Stubbe, M. (1998). Outils de simulation dynamique des réseaux électriques. Techniques De L'Ingenieur, Cahier D 4 120.Paris, France

Mork, B., Gonzalez, F., \& Ishchenko, D. (2007). Hybrid Transformer Model for Transient
Simulation - Part I: Development and Parameters. IEEETrans. on Power Delivery, 22(1), 248-255.

Narang, A., \& Brierley, R. (1994). Topology based magnetic model for steady-state and transient studies for three-phase core type transformers. IEEE Trans. on Power Systems, 9(3), 1337-1349.

\section{SOBRE EL AUTOR}

Leonardo Montealegre Lobo, Ingeniero Eléctrico Master en Ciencias Aplicadas (M.Sc.A). Investigador en Centro de Investigación en Sistemas de Potencia, Instituto Costarricense de Electricidad.

Correo electrónico: Imontealegre@ice.go.cr 